\title{
Compreensão sobre alimentação: visão do portador de diabetes tipo 2
}

\author{
Edelaine Fogaça Avelaneda iD \\ Faculdade de Medicina de Marília - Marília (SP) - Brasil
}

Cláudia Rucco Penteado Detregiachi iD

Faculdade de Medicina de Marília - Marília (SP) - Brasil

Maria Angélica Spadella (iD

Faculdade de Medicina de Marília - Marília (SP) - Brasil

Carlos Aberto Lazarini iD

Faculdade de Medicina de Marília - Marília (SP) - Brasil

Rafaela de Fátima Pereira Baptista (iD

Faculdades Faculdade de Direito da Alta Paulista e Faculdade da Alta Paulista de Tupã - Tupã (SP) - Brasil

Elza de Fátima Ribeiro Higa (iD

Faculdade de Medicina de Marília - Marília (SP) - Brasil

\section{RESUMO}

Objetivo: Analisar se o conhecimento que os portadores de diabetes tipo 2 têm sobre a alimentação contribui com a promoção de sua saúde. Métodos: Pesquisa qualitativa realizada em 2017 por meio de três grupos focais, onde participaram 31 pessoas acometidas pelo diabetes tipo 2, em uma Unidade Básica de Saúde do interior de São Paulo. Os dados obtidos foram interpretados por meio da técnica de análise de conteúdo, modalidade temática. Resultados: A análise apontou três categorias temáticas: Entendimento sobre os alimentos; Alimentos consentidos e Dificuldades relacionadas à alimentação. Conclusão: Constatou-se que a compreensão que os portadores de diabetes do tipo 2 têm sobre sua alimentação pode contribuir parcialmente com a promoção de sua saúde, mas ainda há necessidade de orientações específicas.

Descritores: Diabetes Mellitus Tipo 2; Dieta Saudável; Pesquisa Qualitativa.

\section{INTRODUÇÃO}

A alimentação é compreendida como um fator condicionante da saúde e um direito inerente a todos os seres humanos $^{(1,2)}$. A Lei Brasileira $n^{\circ}$. 11.346, de Segurança Alimentar e Nutricional (LOSAN), em seu $2^{\circ}$ artigo, ressalta que é dever do poder público adotar ações à dignidade da alimentação humana, fazendo-se necessário promover e garantir a segurança alimentar e nutricional de toda população ${ }^{(3,4)}$.

O conhecimento sobre os alimentos e a nutrição está diretamente conexo com a arte de ter saúde por meio da garantia de alimentos com qualidade e quantidade aceitáveis para acolher a necessidade nutricional do ser humano. As opções alimentares são determinadas pelo paladar e pela vontade, influenciado pelas preferências alimentares iniciadas na infância ${ }^{(1,4)}$.

Sendo assim, as escolhas alimentares estão englobadas no contexto em que o sujeito vive, além das aspirações e de todo conhecimento prévio(5). Essas influências da alimentação também estão relacionadas à facilidade de preparo e consumo, ao tempo disponível e ao fator econômico ${ }^{(1,4)}$.

A alimentação é entendida como a terceira necessidade básica humana, ficando atrás da respiração e da ingestão de água. Além de ser imprescindível ao sistema biológico, é considerada também um símbolo no complexo social, político, religioso, ético, sexual e estético. Nesse sentido, vai além do preparo, distribuição e consumo, e completa ainda o que, quando, quanto, onde e com quem se come ${ }^{(4,6)}$.

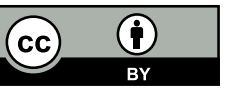


O Guia Alimentar pode servir de referência para a orientação da população brasileira tanto no âmbito individual, como no coletivo, e aponta, em relação às ações de educação, os princípios básicos de uma alimentação adequada e saudável, a fim de garantir a segurança alimentar e nutricional e contribuir com a promoção e a realização do direito humano à alimentação $\operatorname{adequada}^{(4,7)}$.

A conscientização da alimentação saudável deve ser trabalhada com ações de educação alimentar e nutricional, a fim de promover prevenção aos agravos da saúde e melhora na qualidade de vida(4,8).

A alimentação está igualmente regulada pelas regiões geográficas, visto que no Sudeste, por exemplo, são mais consumidos farináceos, batatas, queijos, iogurtes e refrigerantes. Em geral, o consumo de hortaliças e frutas representa a metade das porções recomendadas pelo Guia Alimentar para a População Brasileira, enquanto o consumo de doces e refrigerantes aumenta a cada ano(4,8,9).

A Política Nacional de Alimentação e Nutrição (2013) aponta a alta prevalência de sobrepeso e obesidade entre os brasileiros e, em consequência, um maior número de doenças crônicas não transmissíveis (DCNT). Este aumento está relacionado a um alto consumo de alimentos ultraprocessados com grandes quantidades de açúcar, gorduras e sódio e uma restrição da concentração de nutrientes, vitaminas, minerais, fibras, acompanhados do sedentarismo ${ }^{(4,9)}$.

O mundo passa por uma transição nutricional, fato decorrente de ingestão alimentar inadequada, com alto consumo de alimentos processados e ultraprocessados e o uso abusivo de bebidas alcoólicas, além do tabagismo e da inatividade física ${ }^{(10)}$.

É importante observar que as DCNT são a causa principal de mortalidade e incapacidade antecipada no Brasil. Com elas há um aumento de morbimortalidade que afeta o desenvolvimento social e econômico. Neste contexto, entre as principais doenças estão as cardiovasculares, cânceres, doenças respiratórias crônicas e o diabetes mellitus (DM) ${ }^{(4,11)}$.

A literatura aponta que indivíduos hipertensos e diabéticos apresentam alto consumo de frituras, refrigerantes, carnes gordurosas e adição de sal associado ao baixo consumo de frutas e hortaliças ${ }^{(4,12)}$. Outro estudo realizado em 2013 demonstrou que as mulheres apresentam uma frequência maior no consumo de frutas, frituras, embutidos e refrigerantes comparadas aos homens, e ainda, os habitantes da zona urbana apresentam maior prevalência de consumo de alimentos não saudáveis ${ }^{(4,13)}$.

Estudos indicam que a qualidade dos alimentos tem influenciado no surgimento do diabetes tipo 2, visto que a alta ingestão de ácidos graxos saturados e carboidratos simples e o baixo consumo de cereais integrais, frutas e legumes aumentam o índice de sobrepeso e obesidade e, consequentemente, o risco de desenvolvimento desse tipo de diabetes ${ }^{(4,14)}$.

A incidência de diabéticos vem também aumentando em virtude do crescimento da população e do envelhecimento. A prevalência está se expandindo mais em países de baixa e média renda ${ }^{(15)}$. Em 1980, a prevalência global da diabetes foi de 4,3\%. Já em 2014, foi 9,0\%, mostrando que houve aumento de diabetes em todos os países ${ }^{(16)}$.

Segundo a Federação Internacional de Diabetes, em 2015, havia 415 milhões de pessoas com diabetes no mundo, com maior prevalência no sexo masculino. A estimativa é de que um a cada 11 adultos tem diabetes. $\mathrm{Na}$ América Central e do Sul, em 2015, havia 29,6 milhões de diabéticos. O Brasil ficou em quarto lugar no ranking dos dez principais países cuja população apresenta diabetes em 2015, com 14,3 milhões de diabéticos entre 20 a 79 anos $^{(18)}$. Em 2017, manteve-se o sensível aumento do número de diabéticos no mundo, quando 425 milhões de pessoas eram diabéticas, sendo 327 milhões entre 20 e 64 anos e 98 milhões de 65 a 79 anos. Com 13 milhões de diabéticos nessa faixa etária de 20 a 79 anos, o Brasil ficou novamente em quarto lugar no ranking, atrás da China, da Índia e dos Estados Unidos ${ }^{(17)}$.

A Federação Internacional de diabetes projeta para 2045 um total de 629 milhões de diabéticos no mundo, e 42 milhões na América Central e do Sul. Para a área urbana estimam-se 473 milhões de diabéticos e para a zona rural 156 milhões ${ }^{(17)}$. O DM está entre as principais doenças crônicas no mundo e uma das maiores emergências do século. Assim, é considerada uma epidemia mundial intensa e crescente ${ }^{(17,18)}$.

A Sociedade Brasileira de Diabetes caracteriza o DM como um grupo heterogêneo de distúrbios metabólicos que interfere na ação e secreção da insulina, ocasionando hiperglicemia ${ }^{(19)}$. O diabetes tipo 2 define-se por defeito na ação e/ou secreção da insulina e na regulação da produção hepática de glicose. Na fase pré-clínica da doença, está presente a resistência à insulina, marcada pela redução do efeito estimulante desse hormônio sobre a captação de glicose pelas células periféricas, em especial do músculo e do fígado, e também sobre a inibição da produção hepática de glicose ${ }^{(4,20,21)}$.

Os fatores de risco para diabetes tipo 2 podem estar associados a genéticos, metabólicos, étnicos, histórico familiar e a diabetes gestacional combinada com idade avançada ${ }^{(15)}$. As pessoas portadoras de DM podem ficar impossibilitadas de trabalhar, devido às complicações crônicas, ou permanecem com algum entrave para voltar à vida profissional, causando um custo dessa perda de produtividade e afetando mais a economia do país ${ }^{(16)}$. 
Os custos diretos e indiretos com médicos, medicamentos, hospitais, têm um impacto negativo na economia da saúde ${ }^{(15)}$. Os custos diretos variam entre 2,5 a $15 \%$ do orçamento anual da saúde dos países. Além disso, o indivíduo, a família e a sociedade enfrentam a dor, a ansiedade e a perda da qualidade de vida, custos que não se podem quantificar ${ }^{(22)}$.

Em 2015, o Brasil teve um gasto de 22 bilhões de dólares com despesas de saúde para diabéticos, sendo estimado o custo direto em torno de 3,9 bilhões de dólares ${ }^{(22)}$. De acordo com uma revisão sistemática, estima-se que o custo anual direto do diabetes para o mundo seja superior a $\bigcup \$ 827$ bilhões ${ }^{(16)}$. Esses custos indicam um fardo econômico significativo, afetando também os países de baixa e média renda em seu custeio do sistema de saúde.

Ponderando que a diabetes é uma doença crônica e um problema de saúde mundial, com alta prevalência e incidência, e partindo do desígnio de que a alimentação colabora para o controle da doença, questiona-se: qual o conhecimento dos indivíduos portadores de diabetes tipo 2 sobre sua alimentação? Frente ao exposto, esta pesquisa teve como objetivo analisar se o conhecimento que os portadores de diabetes tipo 2 têm sobre a alimentação contribui com a promoção de sua saúde.

\section{MÉTODOS}

Pesquisa qualitativa que se apresenta pertinente para grupos e segmentos focalizados, quando há necessidade de explorar o universo de significados, motivos, crenças, valores e atitudes sociais. Ela possui instrumentos e técnicas que permitem ao pesquisador alcançar aspectos da subjetividade humana na realidade social(23).

O grupo focal é uma técnica de pesquisa qualitativa que coleta informações por meio das interações grupais, embasada na comunicação e na interação. Seu objetivo prioritário é agrupar conhecimentos delineados a respeito de um assunto peculiar, tendo em vista a obtenção de elementos que possam proporcionar a compreensão do sentido e do significado sobre um determinado fenômeno ${ }^{(24)}$. Desse modo, esta técnica de entrevista coletiva permite que o pesquisador explore as atitudes e opiniões do participante, conhecendo suas percepções por meio das falas, relatos, experiências, vivências e pensamentos a partir de uma problematização ${ }^{(25,26)}$. Além de obter uma maior integração entre os participantes, melhorando os resultados ${ }^{(23)}$.

A pesquisa foi desenvolvida em uma Unidade Básica de Saúde (UBS) de um município do interior do estado de São Paulo, localizado no centro-oeste paulista com uma população estimada de 63.004 habitantes no ano de 2017, sendo 30.409 do sexo masculino e 32.595 do sexo feminino. A população maior de 40 anos representa 13.594 do sexo masculino e 16.310 do sexo feminino, totalizando 29.904 habitantes $^{(27)}$.

Foram convidados 68 pacientes diabéticos tipo 2 para composição dos grupos focais, alfabetizados, em tratamento e com idade superior a 44 anos, de ambos os sexos. Nos dias agendados compareceram 31 deles para as atividades. A dinâmica de coleta de dados foi desenvolvida a partir do esclarecimento quanto aos objetivos e ao modo de participação de cada um, e também sobre quanto ao sigilo das informações prestadas. Todos os participantes assinaram o Termo de Consentimento Livre e Esclarecido (TCLE), antes do início da coleta de dados, seguindo os preceitos para uma pesquisa que envolve seres humanos ${ }^{(28)}$.

No período de 10 de maio a 27 de julho de 2017 foram realizados três grupos focais na própria UBS. Os grupos focais tiveram duração em torno de $60 \mathrm{~min}$, e para preservar o conteúdo original dos depoimentos e garantir fidedignidade foi utilizado um gravador de voz digital a todo o momento da ação ${ }^{(4)}$. A coleta de dados foi realizada por meio de um instrumento constituído por dados sociodemográficos que incluíram: idade, tempo de diagnóstico, sexo, grau de instrução e tipo de tratamento que realiza, acrescidos de três perguntas semiestruturadas direcionadoras da entrevista coletiva: Qual o entendimento que você tem sobre os alimentos? Quais alimentos o (a) senhor (a) acredita que pode comer? Sente dificuldades na alimentação? Quais?

Os grupos tiveram a oportunidade de discutir seus diferentes pontos de vista, o que já proporcionou a possibilidade de aquisição de novos conhecimentos ${ }^{(4)}$.

Para analisar os resultados de pesquisa qualitativa, existem diferentes técnicas e referenciais teóricos, no entanto, a que vem sendo mais utilizada na área da saúde é a Técnica de Análise de Conteúdo na modalidade Temática. A sua utilização exige disciplina, dedicação, rigor científico, pois a análise interpretativa se dá por meio da exatidão da objetividade dos dados obtidos e a fecundidade da subjetividade dos participantes ${ }^{(4,23,29)}$. E os dados obtidos foram interpretados pela Técnica de Análise de Conteúdo, na modalidade Temática segundo $\operatorname{Bardin}^{(30)}$.

Esta análise está estruturada em três etapas: pré-análise, exploração do material e interpretação. A pré-análise consiste em escolher documentos para análise e reaver hipóteses e objetivos iniciais do trabalho. Exploração do material fundamenta-se em analisar e classificar o material para melhor compreensão do texto. A interpretação 
é onde os resultados integrais são submetidos a dados simples ou mais complexos que possibilitam deixar mais evidente as informações obtidas. Com isso, o analista pode sugerir inferências e executar interpretações ${ }^{(23,30)}$.

Assim sendo, foi realizada a leitura flutuante do conjunto, a partir dos dados obtidos nos três grupos focais, seguida pela organização e sistematização das ideias. Na fase de exploração do material, foram codificados os núcleos de sentido das falas dos 31 participantes, tendo em vista a análise categorial ou temática. Em seguida, os dados foram interpretados, levando-se em consideração a pergunta de pesquisa, as respostas obtidas, o referencial teórico e metodológico da pesquisa e o objetivo a ser alcançado ${ }^{(3,30)}$.

No que se refere aos aspectos éticos da pesquisa envolvendo seres humanos, de acordo com a Resolução 466/2012 do Conselho Nacional de Saúde, a pesquisa foi submetida ao Conselho Municipal de Saúde da Secretaria Municipal da Saúde do Município e ao Comitê de Ética em Pesquisa Envolvendo Seres Humanos da Faculdade de Medicina de Marília (FAMEMA) e, autorizada sob o Parecer $n^{0} 1.868 .838^{(28,31)}$. Ainda tendo em vista a preservação do anonimato dos grupos e sigilo dos depoimentos coletados, os participantes foram identificados com a letra $P$, seguida do número equivalente a ordem em que se colocava na conversa. Então, as falas foram identificadas como P1, P2, P3 e, assim, sucessivamente. Após a transcrição, as gravações foram apagadas ${ }^{(4)}$.

\section{RESULTADOS}

Quanto aos dados sociodemográficos dos participantes, obteve-se: faixa etária de 61 a 70 anos; tempo de diagnóstico de 2 a 13 anos; predominância do sexo feminino, do ensino fundamental incompleto e de tratamento do diabetes com medicação oral.

A partir da análise dos dados obtidos nas três perguntas abertas, emergiram três categorias temáticas: 1. Entendimento sobre os alimentos; 2. Alimentos consentidos e 3. Dificuldades relacionadas à alimentação. $\mathrm{Na}$ sequência são apresentadas as categorias e suas falas correspondentes.

\section{Entendimento sobre os alimentos}

Ao debaterem a pergunta: Qual o entendimento que vocês têm sobre os alimentos? Os partícipes apontaram:

"Sustentação no dia-a-dia, senão não consegue sobreviver." (P3)

"Para sobreviver, ter força, não comer exagerado, saber comer." (P9)

"Para sustentar, ficar em pé." (P10; P23)

"O alimento é para repor a energia." (P14; P20)

"Para manter o corpo e a saúde e saber escolher os alimentos." (P5)

Outro aspecto também relatado foi referente ao intervalo entre as refeições:

"Eu aprendi que a cada 3 horas a pessoa tem que se alimentar um pouco, muito pouco." (P1)

"O negócio é comer pouco e de três em três horas." (P4)

"Comer a cada três horas." (P11, P16)

\section{Alimentos consentidos}

Os participantes foram investigados sobre: Quais os alimentos que vocês acreditam que podem comer? Eles, então, relataram:

"Em termo de legumes, pode comer todos, o peixe é bom comer, mas sem excesso. Tem que fugir dos alimentos embutidos e refrigerantes, são moderados." (P3)

"Evitar gordura, fritura." (P19)

"Abacate, aveia, amendoim, iogurte, arroz integral." (P7)

"Arroz integral, pouco feijão, bastante salada, ovos, batata não posso comer, sobe muito." (P15)

"Um pouco de arroz, caldo de feijão, carne todo dia, massa não como muito, não pode comer." (P26)

Foi também possível reconhecer a compreensão dos participantes sobre a quantidade dos alimentos que devem ingerir: 
"O importante é a quantidade." (P1)

"Pode comer de tudo, mas, com moderação." (P2, P14, P18)

"Pode comer de tudo, desde que seja moderado." (P19, P23)

"Tudo deve ser comido moderadamente, o maior problema do diabético é controlar, porque tudo tem que controlar, de tudo um pouco, mas moderado." (P23)

Observou-se também que entre os participantes há certo entendimento de que os alimentos integrais estão relacionados ao controle da glicemia:

“Dar preferência aos integrais, pouco carboidrato." (P11)

"O arroz tem açúcar, as pessoas não sabem, mas tem. Mesmo integral tem que ser pouco." (P1)

"Macarronada é massa." (P22)

\section{Dificuldades relacionadas à alimentação}

E finalmente, quando responderam à pergunta: Vocês sentem alguma dificuldade na alimentação? Os participantes discorreram sobre:

"Em não comer pão, macarrão." (P4)

"Massa, doce, olhar com os olhos e lamber com a testa, eu evito." (P5)

"Não consigo comer sem pão, massa e carne vermelha." (P10)

"Massa, pão, macarrão, muita carne vermelha, refrigerante só final de semana, e a 'coca'." (P6)

"É difícil comer as carnes magras, secas." (P13)

"Dificuldade é de manhã para comer." (P12)

"Comer de 3 em 3 horas." (P11, P16)

\section{DISCUSSÃO}

Na primeira categoria temática da presente pesquisa, os participantes indicaram suas compreensões sobre a alimentação, apontando mais o sentido de sobrevivência como finalidade. A literatura assinala para a necessidade de compreensão também sobre os componentes necessários. Desse modo, apresenta que para promover saúde e bem-estar os alimentos precisam ser ricos em nutrientes e que a alimentação equilibrada e saudável está relacionada à ingestão desses nutrientes de forma variada e como os alimentos são combinados desde o preparo até a ingestão ${ }^{(4,7)}$.

As necessidades fisiológicas do corpo, como crescimento, desenvolvimento, manutenção de tecidos, aumento do sistema imunológico, entre outras, são supridas, a partir da ingestão e da absorção de todos os nutrientes necessários. Recomenda-se que se faça seis refeições distribuídas durante o dia, sendo três refeições principais, como o café da manhã, almoço e jantar e três lanches intermediários, lanche da manhã, lanche da tarde e lanche da noite ${ }^{(4,32)}$. Uma alimentação adequada deve considerar as recomendações de macro e micronutrientes de maneira individualizada, que visa atender as necessidades biológicas no indivíduo ${ }^{(4,19)}$.

Para ter um controle adequado da relação carboidratos e insulina, ou hipoglicemiante oral, deve-se incentivar o diabético a fracionar as refeições, sendo de três em três horas ${ }^{(4,33)}$.

Na segunda categoria do presente estudo, quando os participantes identificaram os alimentos que eles achavam que podiam comer, demonstraram conhecimentos parciais sobre o que é permitido em suas refeições, tanto no que se refere à qualidade, como à quantidade e indicaram suas dificuldades de mudanças de hábitos alimentares, bem como dificuldades para o ajuste das quantidades ingeridas, as quais estruturaram a terceira categoria temática. Nesse sentido, há recomendação de que a alimentação do diabético tipo 2 deve ser variada e equilibrada, atendendo suas necessidades nutricionais, tendo como objetivo a manutenção da glicemia e do peso corpóreo, evitando possíveis complicações de curto e médio prazo ${ }^{(4,34)}$.

É necessária ainda a combinação de todos os grupos alimentares para satisfazer as necessidades nutricionais. Os carboidratos não podem ser restringidos da alimentação, pois haverá um aumento de proteínas e gorduras, que em excesso trazem prejuízos ao controle do diabetes e à saúde ${ }^{(4,35)}$. A ingestão dietética de carboidratos para diabéticos é semelhante à recomendação da população em geral, sendo de 45 a $60 \%$ do valor energético total. Os alimentos ingeridos refletem no controle da sua glicemia, que está associada a uma melhora ou piora do quadro clínico ${ }^{(4,19)}$. 
A quantidade adequada de carboidrato melhora a sensibilidade e a ação da insulina. Assim, para um melhor controle desse processo, pode ser realizada a contagem de carboidrato no plano alimentar diário, considerando todos os grupos alimentares ${ }^{(4,34)}$. Os carboidratos são classificados de acordo com sua composição química em simples e complexos, sendo diferenciados pela digestão e velocidade de absorção, sendo os carboidratos simples mais rápido que os complexos. Os simples são provenientes de açúcar, xarope de milho e glicose. A digestão e absorção destes carboidratos ocorrem de forma mais rápida, provocando elevação abrupta da glicemia ${ }^{(4,32)}$. Já os alimentos integrais, por conterem maior quantidade de fibra em sua composição, tornam mais lento o ritmo da absorção de glicose ${ }^{(4,36)}$, melhorando a glicemia pós-prandial ${ }^{(4,33)}$. As fibras promovem o aumento da saciedade, regulam o funcionamento do intestino e diminuem a concentração plasmática de colesterol ${ }^{(4,32)}$.

Os participantes dos grupos focais da atual pesquisa relataram a exclusão de pão, macarrão, massas e doces, que são classificados como carboidratos simples da rotina alimentar. Contudo, não é necessária a exclusão desses alimentos e, sim, a compreensão de que precisam de um cardápio variado, equilibrado e dentro das necessidades individuais, tornando a alimentação um processo de reeducação alimentar ${ }^{(4,32)}$. Os carboidratos complexos contidos nos grãos integrais, legumes, frutas e produtos lácteos desnatados são recomendados para a alimentação do diabético ${ }^{(4,36)}$. A recomendação de gorduras deve ser individualizada, já que o alto consumo de gorduras saturadas e trans está relacionado ao aumento de peso e às doenças cardiovasculares ${ }^{(4,37)}$.

As principais fontes de ácidos graxos saturados são as carnes gordas, leite integral, óleo de dendê, bacon, torresmo e embutidos e os ricos em gorduras trans são as frituras, tortas industrializadas, bolos, biscoitos recheados e salgados, sorvete de massa e pipoca de micro-ondas ${ }^{(4,19)}$.

As proteínas também devem ser prescritas de forma individualizada, alimentos proteicos sem ou com baixo teor de carboidratos não devem ser utilizados como único alimento no tratamento( ${ }^{(4,38)}$. As principais fontes de proteína animal são carnes magras, leite, queijos e iogurtes com baixos teores de gorduras, e as fontes vegetais incluem soja, leguminosas, cereais integrais e oleaginosas ${ }^{(4,19)}$.

Mesmo sabendo da importância de se alimentar, os diabéticos participantes da pesquisa em questão relataram não terem apetite no período da manhã e dificuldade para se alimentar de três em três horas. A ingestão alimentar deve ser distribuída durante o dia, não se alimentando em excesso e nem realizando jejuns prolongados para manter os níveis glicêmicos controlados, evitando as oscilações que podem causar hiperglicemia ou hipoglicemia ${ }^{(4,32)}$. A necessidade de boa alimentação para manutenção da saúde vem sendo apontada pelos especialistas, tendo em vista o direito humano à alimentação adequada e o controle da obesidade, em particular em pessoas diabéticas ${ }^{(39,40)}$.

\section{CONCLUSÃO}

Considerando o objetivo desta pesquisa foi possível, por meio das técnicas de análise qualitativa, constatar que a compreensão que os portadores de diabetes do tipo 2 têm sobre sua alimentação pode contribuir parcialmente com a promoção de sua saúde, pois os participantes compreendem a importância da alimentação para o dia a dia, mas desconhecem a composição dos alimentos, ingerindo em quantidades excessivas ou restringindo sem necessidade.

O Sistema Único de Saúde possui ações de prevenção e tratamento que são imprescindíveis à educação em saúde para os pacientes diabéticos para que possam apropriar-se de conhecimentos a fim de promover mudanças no tratamento dietético e terem autonomia no cuidado da sua saúde. Contudo, estas ações precisam ser intensificadas, sobretudo, na atenção primária, para melhorar o tratamento dietético à sua realidade e se garantir efetiva promoção da saúde e prevenção de agravos do diabético tipo 2.

\section{AGRADECIMENTOS E CONFLITOS DE INTERESSE}

Autores agradecem a Coordenação de Aperfeiçoamento de Pessoal de Nível Superior (CAPES).

Autores declaram que não houve e não haverá nenhum conflito de interesse.

Este artigo faz parte da Dissertação de Mestrado intitulada: "Cartilha para educação alimentar e nutricional de diabéticos tipo 2", apresentada ao Programa de Mestrado Profissional "Ensino em Saúde", da Faculdade de Medicina de Marília, defendida em 2018. 86 páginas.

\section{REFERÊNCIAS}

1. Alves KP, Jaime PC. The national food and nutrition policy and its dialogue with the national food and nutrition security policy. Cien Saude Colet. 2014;19(11):4331-40. 
2. Pais $S C$, Ferreira PD. A redução das assimetrias sociais no acesso à alimentação e à saúde. Políticas e Práticas. Mais Leitura. 2016.

3. Brasil. Lei n. 11.346, de 15 de setembro de 2006. Cria o Sistema Nacional de Segurança Alimentar e Nutricional-SISAN com vistas em assegurar o direito humano à alimentação adequada e dá outras providências (2006).

4. Avelaneda E, Higa EFR, Detregiachi CRP. Compreensão sobre alimentação: visão do pacientte diabético. Atas CIAIQ 2018. Investigação Qualitativa em Saúde//Investigación Cualitativa en Salud//Volume 2, 2018.

5. Rangel-S ML, Lamego G, Gomes ALC. Alimentação saudável: acesso à informação via mapas de navegação na internet. Physis. 2012;22(3):919-39.

6. Carneiro, H. Comida e sociedade: uma história da alimentação. Rio de Janeiro: Elsevier, 2003.

7. Brasil. Ministério da Saúde. Guia alimentar para a população brasileira. 2a ed. Brasília (DF): Ministério da Saúde; 2014.

8. Fortes LS, Morgado FFR, Almeida SS, Ferreira MEC. Eating behavior and physical activity in adolescents. Rev Nutr. 2013;26:529-37.

9. Brasil. Ministério da Saúde. Política Nacional de Alimentação e Nutrição.Brasília (DF): Ministério da Saúde; 2013.

10. Word Health Organization. Global status report on noncommunicable diseases 2014.Geneva: WHO; 2014.

11. Brasil. Organização Pan Americana da Saúde. Doenças transmissíveis e não-transmissíveis. 2018.

12. Ozcariz SG, Bernardo CO, Cembranel F, Peres MA, Gonzalez-Chica DA. Dietary practices among individuals with diabetes and hypertension are similar to those of healthy people: a population-based study. BMC Public Health. 2015;15:479

13. Destri K, Zanini RdV, Assunção MCF. Prevalência de consumo alimentar entre hipertensos e diabéticos na cidade de Nova Boa Vista, Rio Grande do Sul, Brasil, 2013. Epidemiol Serv Saúde. 2017;26:857-68.

14. Ley SH, Hamdy O, Mohan V, Hu FB. Prevention and management of type 2 diabetes: dietary components and nutritional strategies. Lancet. 2014;383(9933):1999-2007.

15. World Health Organization. Global report on diabetes. Geneva: WHO;2016.

16. Collaboration. NRF. Worldwide trends in diabetes since 1980: a pooled analysis of 751 population-based studies with 4.4 million participants. Lancet. 2016;387(10027):1513-30.

17. International Diabetes Federation. Diabetes atlas. 8th ed. Bruxelas: IDF; 2017.

18. Iser BPM, Stopa SR, Chueiri PS, Szwarcwald CL, Malta DC, Monteiro HOC, Duncan BB, Schmitd MI. Prevalência de diabetes autorreferido no Brasil: resultados da Pesquisa Nacional de Saúde 2013. Epidemiol Serv Saúde. 2015;24:305-14.

19. Sociedade Brasileira de Diabetes. Diretrizes da Sociedade Brasileira de Diabetes: 2019-2020. Rio de Janeiro (RJ): Clannad Editora Científica;2020.

20. Hall JE. Tratado de Fisiologia Médica. In: Elsevier, editor. Insulina, Glucagon e Diabetes Mellitus.12 ed;2011.

21. Sociedade Brasileira Diabetes. Diretrizes da Sociedade Brasileira de Diabetes:2017-2018. São Paulo (SP): SBD;2017.

22. Sociedade Brasileira de Diabetes. Diretrizes da Sociedade Brasileira de Diabetes: 2015-2016. Rio de Janeiro (RJ): AC Farmacêutica; 2016.

23. Minayo C. O desafio do conhecimento: pesquisa qualitativa em saúde. 13a ed. Petropolis (RJ): Vozes; 2013.

24. Kinalski DDF, Paula CCD, Padoin SMM, Neves ET, Kleinubing RE, Cortes LF. Focus group on qualitative research: experience report. Rev Bras Enferm. 2017;70:424-9.

25. Silva MG, Fernandes JD, Rebouças LC, Rodrigues GRS, Teixeira GA, Silva RMO. Publicações que utilizaram o grupo focal como técnica de pesquisa: o que elas nos ensinam? Cienc Cuid Saude. 2013; 12(2):398-406. 
26. Medeiros HP, Teixeira E. Metodologia da pesquisa para a enfermagem e saúde: Resenha de livro. Rev Bras Enferm. 2016; 69:1000-1.

27. Fundação Sistema Estadual de Análise de Dados. Projeções populacionais: projeção de população residente em $1^{\circ}$ de julho, Tupã 2016. [Internet]. São Paulo (SP): SEAD. 2016. [Acesso em 2017 Out 10]. Disponível em: http://produtos.seade.gov.br/produtos/projpop/

28. Brasil. Ministério da Saúde. Conselho Nacional da Saúde. Resolução n 510 , de 7 de abril de 2016. Aprova as diretrizes e normas regulamentadoras de pesquisas envolvendo seres humanos. Diário Oficial da União, Brasília, 24 maio. 2016, Seção 1, p. 44. Disponível em: http://bvsms.saude.gov.br/bvs/saudelegis/cns/2016/ res0510_07_04_2016.html>

29. Silva AH, Fossá MIT. Análise de conteúdo: exemplo de aplicação da técnica para análise de dados qualitativos. Qualit@s Rev Eletrônica [Internet]. 2015 [Acesso em: 2018 Abr 10];17:1-14. Disponível em: http://oficinas.incubadora.ufsc.br/index.php/Lucasfranco/article/view/2336/2155

30. Bardin L. Análise de conteúdo. São Paulo: Edições 70. 2012.

31. Brasil. Ministério da Saúde, Conselho Nacional da Saúde. Resolução n 466 , de 12 de dezembro de 2012. Aprova as diretrizes e normas regulamentadoras de pesquisas envolvendo seres humanos [Internet]. Diário Oficial da União, Brasília (DF); 13 jun 2013; Seção 1:59. Disponível em: http://bvsms.saude.gov.br/bvs/ saudelegis/cns/ 2013/res0466_12_12_2012

32. Molmelstet KC, Fanton S, Bertoncini JH, Silva CRLD, Vargas DM, Campanella LCA. Conhecimento nutricional de uma equipe multiprofissional que atende pessoas com diabetes mellitus na atenção básica. Rev APS. 2016;19(1):31-8.

33. Dworatzek PD, Arcudi K, Gougeon R, Husein N, Sievenpiper JL, Williams SL. Nutrition therapy. Can J Diabetes. 2013;37(Suppl 1):S45-55.

34. American Diabetes Association. Standards of Medical Care in Diabetes 2015. Diabetes Care. 2015;38 (Suppl 1):S20.

35. Souto DL, Rosado EL. Contagem de carboidratos no Diabetes Melito: abordagem teórica e prática. 2a ed.Rio de Janeiro (RJ): Rubio;2018.

36. Silva SMCS, Mura, JDP. Tratado de alimentação e dietoterapia. 3a ed. São Paulo (SP): Roca;2016.

37. American Diabetes Association. Diabetes care in the hospital. Diabetes Care. 2017;40(Suppl 1):S120-7.

38. Gower BA, Goss AM. A lower-carbohydrate, higher-fat diet reduces abdominal and intermuscular fat and increases insulin sensitivity in adults at risk of type 2 diabetes. J Nutr. 2015;145(1):177S-83S.

39. Cardoso BEP, Campelo IM, Aguiar EMG, Paz SMRS, Rocha LRS, Santos MM Social participation of food and nutrition security counselors. Rev Bras Promoç Saúde, 2018;31(3):1-8.

40. Godoy AR, Adami FS. Estado nutricional e qualidade de vida em adultos e idosos com depressão. Rev Bras Promoç Saúde, 2019;32:7354.

\section{Endereço do primeiro autor:}

Edelaine Fogaça Avelaneda

Rua José de Grande, 332

Bairro: Jardim Parati

CEP: 17519-470 - Marília SP - Brasil

E-mail: edelaineavelaneda@gmail.com

\section{Endereço para correspondência:}

Elza de Fátima Ribeiro Higa

Rua José de Grande, 332

Bairro: Jardim Parati

CEP: 17519-470 - Marília SP - Brasil

E-mail: hirifael@gmail.com 